\title{
Effect of Different Pulse Durations on the Efficacy of Long-Pulsed Alexandrite-Assisted Hair Removal; A Split-Face Comparison Study
}

\author{
Pedram Noormohammadpour ${ }^{1,2}{ }^{\complement}$, Amir Hooshang Ehsani ${ }^{1,2}{ }^{\complement}$, Farshad Farnaghi ${ }^{1{ }^{12}}$, Hassan Seirafi ${ }^{1,2}$, Seyed \\ Alamdar Bagherinia ${ }^{1,2}$, Parvaneh Hatami ${ }^{1,2^{*}}{ }^{\star}$, Azadeh Goodarzi ${ }^{3 \oplus}$, Zeinab Aryanian ${ }^{1,4^{*}}$ \\ 'Autoimmune Bullous Diseases Research Center, Tehran University of Medical Sciences, Tehran, Iran \\ ${ }^{2}$ Department of Dermatology, School of Medicine Razi Hospital, Tehran University of Medical Sciences, Tehran, Iran \\ ${ }^{3}$ Department of Dermatology, School of Medicine, Rasoul Akram Hospital, Iran University of Medical Sciences, Tehran, \\ Iran \\ ${ }^{4}$ Department of Dermatology, Babol University of Medical Sciences, Babol, Iran
}

\begin{abstract}
*Correspondence to Zeinab Aryanian, Autoimmune Bullous Diseases Research

Center, Razi hospital,Tehran University of Medical Sciences, Tehran, Iran. Email: aryanian@ sina.tums.ac.ir, z_aryanian@ yahoo.com; Parvaneh Hatami, Autoimmune Bullous Diseases Research Center, Razi

hospital,Tehran University of Medical Sciences, Tehran, Iran Tel: +989124848444, Email: p_hatami2001@yahoo.com
\end{abstract}

Published online May 20, 2021

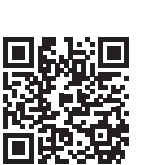

\section{Introduction}

Unwanted hair growth which is seen in some pathologic situations such as hypertrichosis or hirsutism is one of the most common complaints among patients of dermatology clinics. Over the past decade, hair removal lasers have been used widely for long-term hair reduction and their technology has advanced very fast. In fact, the invention of these instruments has been a revolution in the hair removal industry, with many different laser systems currently available. ${ }^{1-3}$

Melanin, the natural chromophore of these types of lasers, absorbs the laser light and transforms energy into heat. The rate and extent of this heat are determined by the fluence and the duration of exposure. ${ }^{4}$ Each of the pulsed light sources generates different pulse durations, spot sizes and fluences that provide varying clinical responses. ${ }^{5}$ An astute clinician with a precise selection of these parameters can produce desired tissue injuries confined to the chromophore. The main targets regarding hair removal laser treatment appear to be the dermal papilla and the bulge. ${ }^{6}$

Selective thermal damage of these targets happens when sufficient energy density at a given wavelength which is superiorly absorbed by the chromophore, is released at a time period not longer than the thermal relaxation time (TRT) of the target.

Many studies have been conducted to examine the impact of different spot sizes and fluences on clinical improvement and the side effect profile of various hair removal lasers, ${ }^{7-9}$ but as far as we know, there are not too many studies for the examination of the outcome of treatment using different pulse durations, although this parameter has been shown to be important. The pulse duration (or pulse width) must be long enough for the epidermal melanin to throw away the heat and therefore be safe regarding thermal injury, but short enough to keep adequate energy in the hair follicle to destroy it.

The TRT for the epidermis is determined to be 3-10 milliseconds in previous studies, while this value for the hair follicle, depending on its diameter, is about 10-40 
milliseconds. ${ }^{10}$

Therefore, rationally, the ideal should be something between these two values.

This prospective study aimed to explore the effect of different pulse durations on the clinical efficacy and the adverse effects of laser-assisted hair removal using the 755 $\mathrm{nm}$ alexandrite laser system.

\section{Materials and Methods Study Design}

This was a single-centre, prospective clinical study that was conducted for 9 months from September 2018 to May 2019 at Razi hospital, the main dermatology-specific teaching hospital of our country.

A total of 50 female subjects with facial hirsutism and Fitzpatrick skin types II-IV were enrolled.

The patients with the following features were excluded from the study: history of hair removal treatment for their facial hirsutism, severe photosensitivity, keloidal or hypertrophic scar tendency, recent sun exposure, immunosuppression and pregnancy.

After an explanation of the study to participants, informed written consent was signed by each patient. Then, their demographic data were collected.

\section{Clinical Evaluation and Setting}

For the purpose of the study, a detailed history in regards to the onset of hirsutism and its rate of progression, a family history, a past medical history, a drug history and any sign of polycystic ovary syndrome (PCOS) or other forms of hormonal dysfunction were obtained from each patient in the first session. Then, areas of unwanted hair growth were identified and marked into two $2 \times 2 \mathrm{~cm}$ facial regions on each side of the face. A digital camera (canon power shot SX-200) captured images at the baseline and 1 month after the last laser treatment utilizing standard equipment and lightening.

The patients were instructed not to shave or remove hair from the treatment area for at least 1 week prior to the treatment and also asked to avoid waxing, threading, bleaching or plucking hairs for at least one month before the initiation of the study. No special preoperative topical skin lightening agents or other skin care products were prescribed.

On each treatment session, the face was rinsed with water and dried. No topical anesthesia was used. Hairbearing quadrants were trimmed or shaved to obtain a hair shaft shorter than $1 \mathrm{~mm}$ above the skin surface.

The treatment was initiated with safe parameters in a conservative manner, especially in darker skin types: Using the manufacturer's -recommended methods and settings, one side of the face of each patient (randomly determined) was treated with 10-millisecond pulse duration and the other side with 3-millisecond pulse duration with an alexandrite laser (Gentlemax Pro ${ }^{\oplus}$, Candela $^{\mathrm{TM}}$, USA).
Other parameters (Fluence, DCD at 40/30, spot size of $18 \mathrm{~mm}$ and repetition rate of $1 \mathrm{~Hz}$ ) were the same for both sides of the face.

The treatment started with $14 \mathrm{~J} / \mathrm{cm}^{2}$ for skin type II, 13 $\mathrm{J} / \mathrm{cm}^{2}$ for skin type III and $12 \mathrm{~J} / \mathrm{cm}^{2}$ for skin type IV. In the next sessions, fluence decreased or increased by $1 \mathrm{~J} / \mathrm{cm}^{2}$ according to the presence of side effects as well as patient tolerance. We achieved our desired results with fluences ranging from 14 to $18 \mathrm{~J} / \mathrm{cm}^{2}$.

The subjects underwent three treatment sessions at regular time intervals of 5 weeks.

The number of regrowing hairs was counted by a physician and the clearance rates were determined with regards to the presence of hair reduction by the visual analogue scale (VAS) as follows:

Patients without any change, and a clearance rate of less than $25 \%, 25 \%$ to $<50 \%, 50 \%$ to $75 \%$ and more than $75 \%$.

An effective treatment was defined as achieving a hair reduction of more than $50 \%$.

The occurrence of any adverse effects including erythema, hypo- or hyperpigmentation and scarring was documented as well.

Statistical analysis was performed using SPSS, version 23. A $P$ value $<0.05$ was considered significant. Chi-square and McNemar's tests were used to show correlations.

\section{Results}

\section{Patient Characteristics}

Fifty female patients with facial hirsutism, whose ages ranging from 20 to 59 years (mean: 34.7 years), completed the study. $6 \%(n=3)$ of the patients had skin type II, $64 \%$ $(n=32)$ had skin type III and $30 \%(n=15)$ had skin type IV. Sixteen percent of the subjects had a previous history of PCOS and $84 \%$ did not have any history of PCOS or other hormonal disorders. None of the patients discontinued the treatment over the course of the study.

On the basis of hair density recorded at the beginning and one month after the last treatment session, we found that $96 \%$ of the subjects showed some degrees of hair reduction with 3 milliseconds pulse duration and 94\% with 10 milliseconds pulse duration (Table 1). Effective treatment (defined as clearance rate higher than 50\%) was seen in $56 \%$ of patients with both pulse durations (Figure 1).

Table 1 shows the number of patients in each group (based on the rate of hair reduction) with 3 and 10

Table 1. The Clearance Rate With 3 and 10 Milliseconds Pulse Durations

\begin{tabular}{lccc}
\hline Clearance Rate (\%) & With 3 msec & With 10 msec & P Value \\
\hline $0 \%$ & $4 \%$ & $6 \%$ & 1.000 \\
$0-25 \%$ & $6 \%$ & $6 \%$ & 1.000 \\
$25-50 \%$ & $34 \%$ & $32 \%$ & 1.000 \\
$50-75 \%$ & $42 \%$ & $40 \%$ & 1.000 \\
$75-100 \%$ & $14 \%$ & $16 \%$ & 1.000 \\
\hline
\end{tabular}




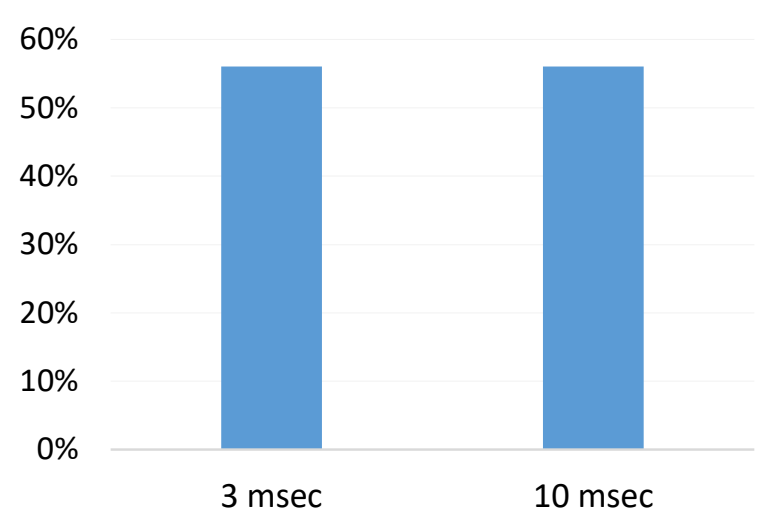

Figure 1. The Clearance Rate $>50 \%$ with 3 and 10 Milliseconds Pulse Durations 1 Month After the Treatment ( $P$ value: 1.000)

milliseconds pulse duration (PD) respectively. The difference between 3 and 10 milliseconds pulse durations on each group was not statistically significant $(P$ value for each group: 1.000).

We also compared the efficacy of the treatment with these two pulse durations in PCOS versus nonPCOS subjects. Using the chi-square test, we found no significant difference between PCOS and non-PCOS patients with 3 and 10 milliseconds ( $P$ value: 0.441 and 1.000 respectively).

Based on Fitzpatrick skin phototypes, we found that the clearance rate $>50 \%$ was seen in $40 \%$ of the subjects with skin type IV and $42 \%$ of the subjects with skin type II or III with a 3 milliseconds pulse duration $(P$ value $=0.851)$ (Table 2).

With a 10 milliseconds pulse duration, the clearance rate higher than $50 \%$ was achieved in $33 \%$ of the subjects with skin type IV and $42 \%$ with skin type II or III ( $P$ value $=0.529$ ). There were no adverse events noted during the course of the study.

\section{Discussion}

One of the most effective methods for delaying the growth of unwanted hairs is the use of a $755 \mathrm{~nm}$ alexandrite laser. ${ }^{5}$ The selective photothermolysis principle predicates the selective thermal damage of a chromophore by light and it is the mainstay of a safe and effective treatment using different types of lasers. Therefore, first of all, we should determine the important targets, and regarding hair removal, the main target sites appear to be the germinative cells of the hair, matrix and papilla or bulb and/or the follicular stem cells detected next to the erector pili muscle or bulge. ${ }^{11}$ Laser irradiation with appropriate fluence, released at a given wavelength and within a time duration not longer than the TRT of the target, preferentially absorbed by the target chromophore. At longer pulse durations, greater tissue injury must be expected with wide targets including hair follicles, because there is enough time for the laser beam to be absorbed by the targets. ${ }^{5}$ So theoretically, for the best outcome, pulse duration would be in a range between the TRT for the epidermis (3-10 milliseconds) and that for hair follicles (10-100 milliseconds for hair follicles 100-300 $\mu \mathrm{m}$ in diameter). ${ }^{12,13}$

The alexandrite laser is available with a pulse duration from 2 to 40 milliseconds depending on the manufacturer. In our study, the pulse durations were 3 and 10 milliseconds which are at or near the TRT of the epidermis which let the epidermis be spared during air cooling.

Several studies show the effectiveness of the alexandrite laser with different pulse durations in hair removal. A study by Finkel et $\mathrm{al}^{14}$ showed a good outcome in the treatment of hypertrichosis on the whole body with the long-pulsed 2 milliseconds alexandrite laser. In a similar study by Raulin and Greve, ${ }^{15}$ the alexandrite laser with longer pulse widths was used and proved to be a good method of treating unwanted hairs. Their patients achieved an average clearance rate of $75 \%$ after an average of 8 treatments of facial hirsutism. However, just a few studies have compared the effect of different pulse durations on the effectiveness of the alexandrite laser. To the best of our knowledge, this is the first splitface controlled clinical trial that evaluated the effect of increasing the pulse duration on alexandrite laser efficacy in Asian skin and also investigated the impact of having a PCOS history on the treatment outcome.

According to theoretical considerations, an alteration in pulse width should change both results and side effects. For instance, the elongation of PD might induce less destruction to the epidermal melanosomes in contrast with shorter PDs, because small targets such as epidermal melanosomes are more vulnerable to damage by short pulses. Ataie-Fashtami et al simulated the effect of various laser parameters on heat distribution and the thermal damage pattern of hair and epidermis and found that longer PDs were associated with less epidermal thermal damage, but an excessive increase in PD was accompanied by unwanted thermal damage to the adjoining dermis. They suggested using a diode laser with longer PDs up to 400 milliseconds in order to reach the best clinical results along with avoiding side effects. ${ }^{16}$ In regard to the relationship between $\mathrm{PD}$ and clinical results, contrary to theoretical considerations, our study showed that the prolongation of PD on the long-pulsed alexandrite laser does not necessarily lead to better results. In other words, the impact of pulse duration on clinical outcome

Table 2. The Efficacious Treatment Rate on Each Pulse Duration According to Skin Type

\begin{tabular}{lccc}
\hline & \multicolumn{2}{c}{ Clearance Rate $>\mathbf{5 0} \%$} & \multirow{2}{*}{ P Value } \\
\cline { 2 - 3 } & Skin Type II or III & Skin Type IV & \\
\hline PD: $10 \mathrm{msec}$ & $42 \%$ & $40 \%$ & 0.851 \\
PD: $3 \mathrm{msec}$ & $42 \%$ & $33 \%$ & \\
\hline
\end{tabular}


of hair removal treatment with alexandrite laser may be less than expected before. In fact, the results of our study are consistent with some previous studies using different PDs. Nanni et al examined the effect of PD on the clinical results of the long-pulsed alexandrite laser on hair removal of different regions of the body using 5,10 and 20 milliseconds pulse widths. Their results revealed no significant difference in the clinical improvement rate and side effects. ${ }^{5}$

In another study by Boss et al, who used 2 and 20 milliseconds PDs. Similar observations were seen in different body areas after a 6-month follow-up. ${ }^{17}$ In contrast, McDaniel et al found better results by longer pulse durations. ${ }^{18}$

Based on our results, hair regrowth at one month after the last session of laser therapy was not significantly different by raising the PD from 3 to 10 milliseconds. The fact that all PDs resulted in equivalent hair reduction may be a result of a short follow-up period, Thus, further studies with longer follow-up durations and a larger sample sizes are encouraged to find out the exact effect of $\mathrm{PD}$ on the efficacy of hair removal lasers.

Although we could not detect any distinction between two different pulse widths (3 and 10 milliseconds) used in this study in terms of the safety profile, theoretically, the 10 milliseconds PD would be awaited to induce fewer epidermal injuries because of its relative sparing of tiny epidermal melanosomes. ${ }^{19}$ It should be borne in mind that a large part of this study was conducted during autumn and winter, which can be an important factor in a reduction in the incidence of sun exposure related side effects.

Few studies have reported a decrease in postinflammatory hyperpigmentation after using the 20 milliseconds PD due to this epidermal sparing phenomenon at longer pulse durations. ${ }^{19}$

An interesting finding of this study was the absence of any difference in the hair reduction regarding the history of PCOS in our patients. McGill et al demonstrated that women with PCOS may benefit less than expected from laser hair removal, ${ }^{20}$ but a recent study showed the effectiveness of a $755 \mathrm{~nm}$ alexandrite laser with 3 milliseconds pulse duration in contrast to intensed pulse light (IPL) in this group of women. ${ }^{21}$

Further studies should also address the question of whether the positive history of PCOS can affect the clinical outcome of hair removal treatment using different types of lasers.

Finally, there was no significant difference in clinical response regarding skin type that is congruent with previous studies. ${ }^{2}$

In conclusion, the results of this study further support the effective and safe use of a $755 \mathrm{~nm}$ alexandrite laser with both 3 and 10 milliseconds pulse durations for hair removal in patients with II-IV skin phototypes.

Despite the success of this study, some limitations must be considered, including a lack of devices for objective measurement of clinical improvement as well as a limited number of treatment sessions due to some financial issues regarding the research budget. Moreover, despite the fact that the number of participants in our study was comparable to other similar ones, enrolling more patients makes it easier to reach a more reliable result in regards to both clinical efficacy and the occurrence of adverse effects.

Given these limitations, further investigations with larger sample sizes, longer follow-ups, using devices to quantify the thickness of the hair or even histologic examination of treated sites are suggested to fully understand the impact of pulse width on laser-assisted hair removal.

\section{Ethical Considerations}

The study protocol was approved by a relevant ethics committee and was registered on the Iranian Registry of Clinical Trials (identifier: IRCT20191228045917N1).

\section{Conflict of Interests}

The authors declare that there is no conflict of interest regarding the publication of this article.

\section{Acknowledgement}

This study as an internship research thesis was performed at the Autoimmune Bullous Diseases Research Center.

\section{References}

1. Nanni CA, Alster TS. A practical review of laser-assisted hair removal using the Q-switched Nd:YAG, long-pulsed ruby, and long-pulsed alexandrite lasers. Dermatol Surg. 1998;24(12):1399-405. doi: 10.1111/j.1524-4725.1998. tb00022.x.

2. Tulpule MS, Bhide DS, Bharatia P, Rathod NU. $810 \mathrm{~nm}$ diode laser for hair reduction with Chill-tip technology: prospective observational analysis of 55 patients of Fitzpatrick skin types III, IV, V. J Cosmet Laser Ther. 2020; 22(2):65-69. doi: 10.1080/14764172.2020.1726961.

3. Załęska I, Atta-Motte M. Aspects of diode laser (805 nm) hair removal safety in a mixed-race group of patients. J Lasers Med Sci. 2019; 10(2):146-153. doi: 10.15171/ jlms.2019.23.

4. Pai GS, Bhat PS, Mallya H, Gold M. Safety and efficacy of low-fluence, high-repetition rate versus high-fluence, low-repetition rate $810-\mathrm{nm}$ diode laser for permanent hair removal--a split-face comparison study. J Cosmet Laser Ther. 2011;13(4):134-7. doi: 10.3109/14764172.2011.594057.

5. Nanni CA, Alster TS. Long-pulsed alexandrite laserassisted hair removal at 5, 10, and 20 millisecond pulse durations. Lasers Surg Med. 1999;24(5):332-7. doi: 10.1002/ (sici)1096-9101(1999)24:5<332::aid-lsm3>3.0.co;2-2.

6. Ort RJ, Anderson RR. Optical hair removal. Semin Cutan Med Surg. 1999;18(2):149-58. doi: 10.1016/s10855629(99)80039-x.

7. Bäumler W, Scherer K, Abels C, Neff S, Landthaler M, Szeimies RM. The effect of different spot sizes on the 
efficacy of hair removal using a long-pulsed diode laser. Dermatol Surg. 2002;28(2):118-21. doi: 10.1046/j.15244725.2002.01117.x.

8. Goldberg DJ, Silapunt S. Histologic evaluation of a millisecond Nd:YAG laser for hair removal. Lasers Surg Med. 2001;28(2):159-61. doi: 10.1002/lsm.1033.

9. Jo SJ, Kim JY, Ban J, Lee Y, Kwon O, Koh W. Efficacy and safety of hair removal with a long-pulsed diode laser depending on the spot size: a randomized, evaluatorsblinded, left-right study. Ann Dermatol. 2015; 27(5):51722. doi: 10.5021/ad.2015.27.5.517.

10. Ross EV, Ladin Z, Kreindel M, Dierickx C. Theoretical considerations in laser hair removal. Dermatol Clin. 1999;17(2):333-55. doi: 10.1016/s0733-8635(05)70091-7.

11. Lyle S, Christofidou-Solomidou M, Liu Y, Elder DE, Albelda S, Cotsarelis G. The C8/144B monoclonal antibody recognizes cytokeratin 15 and defines the location of human hair follicle stem cells. J Cell Sci. 1998;111 (Pt 21):3179-88.

12. Nanni CA, Alster TS. Optimizing treatment parameters for hair removal using a topical carbon-based solution and 1064-nm Q-switched neodymium:YAG laser energy. Arch Dermatol. 1997;133(12):1546-9.

13. Bhat YJ, Bashir S, Nabi N, Hassan I. Laser treatment in hirsutism: an update. Dermatol Pract Concept. 2020;10(2):e2020048. doi: 10.5826/dpc.1002a48

14. Finkel B, Eliezri YD, Waldman A, Slatkine M. Pulsed alexandrite laser technology for noninvasive hair removal. J Clin Laser Med Surg. 1997;15(5):225-9. doi: 10.1089/ clm.1997.15.225.

15. Raulin C, Greve B. Temporary hair loss using the long- pulsed alexandrite laser at 20 milliseconds. Eur J Dermatol. 2000;10(2):103-6.

16. Ataie-Fashtami L, Shirkavand A, Sarkar S, Alinaghizadeh M, Hejazi M, Fateh M, et al. Simulation of heat distribution and thermal damage patterns of diode hair-removal lasers: an applicable method for optimizing treatment parameters. Photomed Laser Surg. 2011; 29(7):509-15. doi: 10.1089/ pho.2010.2895.

17. Boss WK Jr, Usal H, Thompson RC, Fiorillo MA. A comparison of the long-pulse and short-pulse Alexandrite laser hair removal systems. Ann Plast Surg. 1999; 42(4):3814. doi: 10.1097/00000637-199904000-00006.

18. McDaniel DH, Lord J, Ash K, Newman J, Zukowski M. Laser hair removal: a review and report on the use of the long-pulsed alexandrite laser for hair reduction of the upper lip, leg, back, and bikini region. Dermatol Surg. 1999;25(6):425-30. doi: 10.1046/j.1524-4725.1999.08118.x.

19. Nanni CA, Alster TS. Laser-assisted hair removal: side effects of Q-switched Nd:YAG, long-pulsed ruby, and alexandrite lasers. J Am Acad Dermatol. 1999; 41 (2 Pt 1):165-71. doi: 10.1016/s0190-9622(99)70043-5.

20. McGill DJ, Hutchison C, McKenzie E, McSherry E, Mackay IR. Laser hair removal in women with polycystic ovary syndrome. J Plast Reconstr Aesthet Surg. 2007;60(4):426-31. doi: 10.1016/j.bjps.2006.11.006.

21. McGill DJ, Hutchison C, McKenzie E, McSherry E, Mackay IR. A randomised, split-face comparison of facial hair removal with the alexandrite laser and intense pulsed light system. Lasers Surg Med. 2007;39(10):767-72. doi: 10.1002/ lsm.20584. 